\title{
Metabolic syndrome and insulin resistance in an urban and rural adult population in Sri Lanka
}

\author{
R Jayatissa ${ }^{1}$, Y Yamori ${ }^{2}$, A H De Silva ${ }^{3}$, M Mori $^{2}$, S Gunawardana ${ }^{1}$, U De Silva ${ }^{1}$, J M Ranbanda $^{1}$ \\ Sri Lanka Journal of Diabetes, Endocrinology and Metabolism 2014; 4: 9-16
}

\begin{abstract}
Background: To assess the prevalence of metabolic syndrome and insulin resistance in an urban and rural population in Sri Lanka.

Methods: A cross sectional study conducted in 3 health areas selected randomly, one in rural and 2 in urban. In each sector, 180 adults between 30-59 years equally from both sexes were included. Blood pressure, weight, height and waist circumference were measured in each participant. Fasting blood samples were taken to assess plasma glucose (FPG), triglyceride (TG), high density lipoprotein (HDL), insulin and $\mathrm{HbA1C}$. Insulin resistance was detected by the homeostasis model assessment method (HOMA-IR).

Results: From total 345 participants, 46.1\% were men and 52.9\% women. Prevalence of metabolic syndrome was $25.8 \%$ (23.9\% in men and $27.4 \%$ in women; $\mathrm{P}=0.27$ ). Prevalence of each component of MetS in studied population was: $62.9 \%$ for central obesity, 28.1\% for high TG, 35.9\% for low HDL, 40\% for high BP, $18.8 \%$ for high FPG, 3.9\% for high HbA1C, 44.1\% for overweight and 9.3\% for obesity. Hypeinsulinaemia and insulin resistance was $26.9 \%$ and $22.3 \%$ respectively. Age, high BMI, waist circumference, FPG, TG, BP, HbA1C, insulin and low HDL significantly higher with subjects with MetS $(P=0.000)$. Hyperinsulinaemia and insulin resistance was significantly higher in rural sector, obese, centrally obese, high BP and high TG subjects.
\end{abstract}

Conclusion: One fourth of study population had metabolic syndrome, hyperinsulinaemia and insulin resistance. Metabolic syndrome was strongly associated with hyperinsulinaemia and insulin resistance. Need urgent action to reduce risk in developing type-2 diabetes and cardiovascular diseases in this population.

Keywords: metabolic syndrome, insulin resistance, hyperinsulinaemia

\section{Introduction}

Metabolic syndrome (MetS) is a complex disorder with high socioeconomic cost that is considered a worldwide epidemic. The metabolic syndrome is a cluster of the most dangerous heart attack risk factors: diabetes and raised fasting plasma glucose, abdominal obesity, high cholesterol and high blood pressure (1-3). Although there are various definitions for metabolic syndrome, recent review concluded that the definition incorporating International Diabetes Federation (IDF) and American Heart Association / National Heart, Lung and Blood Institute (AHA / NHLBI) is the most suitable for practical use in clinical medicine (1).

People with metabolic syndrome are twice as likely to die from and three times as likely to have heart attack or stroke and have a fivefold greater risk of developing type 2 diabetes compared with people without the syndrome (4). In addition, there is a five fold greater risk of developing type 2 diabetes. MetS would add to the 230 million people worldwide who already have diabetes, one of the most common chronic diseases worldwide and the fourth or fifth leading cause of death in the developed world. The clustering of cardiovascular disease (CVD) risk factors that typifies the metabolic syndrome is now considered to be the driving force for a new CVD epidemic.

Insulin resistance is associated with multiple risk factors for atherosclerosis, including hypertension, dyslipidemia, and glucose intolerance or type 2 diabetes mellitus. Clustering of these risk factors in subjects with

${ }^{1}$ Department of Nutrition, Medical Research Institute, Ministry of Health, Colombo, ${ }^{2}$ Mickagowa University, Japan, ${ }^{3}$ Department of Health, Western Province, Sri Lanka. 
obesity has been called "syndrome X" (5), "deadly quartette" (6), "insulin resistance syndrome" (7), or "visceral fat obesity syndrome" (8). Hyperinsulinemia is a good marker of insulin resistance in subjects without significant hyperglycemia and is used for epidemiologic studies.

Insulin resistance and central obesity are considered as significant underlying factors of MetS, which predisposes the global epidemics of type 2 diabetes and cardiovascular diseases (CVD). Genetics, physical activity, ageing, a pro-inflammatory state and hormonal changes may also have a causal effect, but the role may depend on ethnicity (9).

It is estimated that around 20-25 percent of the world's adult population have metabolic syndrome (4). Prevalence of metabolic syndrome has been reported to be between $12.8 \%$ to $41.1 \%$ in different parts of the world $(10,11)$. Studies in Sri Lanka showed that the prevalence of metabolic syndrome was between $27.1 \%$ to $29.5 \%(12,13)$. Many factors including: female gender, increasing age, urban living, higher socio-economic status and physical inactivity were reported as important associated factors (12). According to the World Health Organisation, Non Communicable Diseases (NCDs) are estimated to account for $65 \%$ of all deaths in Sri Lanka, accounting $30 \%$ of CVD and $4 \%$ of diabetes. About $39 \%, 9 \%$, $22 \%$ and $5 \%$ of adults are suffering from raised blood pressure, raised blood sugar, overweight and obesity respectively (14).

However, early identification of patients and treatment with appropriate medical and educational programs can be an effective step in controlling and reducing the incidence of metabolic syndrome and in turn cardiovascular diseases and diabetes. In Sri Lanka, last national survey was conducted in 2005-2006. The aim of this study was to assess the recent prevalence of MetS and insulin resistance in an urban and rural population in Sri Lanka to implement suitable interventions to prevent development of diabetes and cardiovascular diseases.

\section{Materials and methods}

The study population was healthy adults (both male and female) between $30-59$ years. Calculated sample size was 360;180 from each urban and rural sector considering $27 \%$ of the prevalence of MetS, $95 \%$ of precision, 1.1 design effect and $10 \%$ non-response rate. A total of 360 adults; 180 from each sector were included in the study. Three Medical Officer of Health (MOH) areas were selected from the Western Province; 2 urban and 1 rural. Ten Public Health Midwives (PHM) were randomly selected from each $\mathrm{MOH}$ area to draw the sample. Each selected PHM in the urban $\mathrm{MOH}$ areas and rural $\mathrm{MOH}$ areas were asked to recruit 9 and 18 healthy adults respectively within the PHM area, starting from randomly selected one starting point, equal numbers from male and female, from each age groups (30-39, 40-49 and 50-59). All the selected adults were asked to attend the relevant $\mathrm{MOH}$ office on the predetermined date. Body weight, height and waist circumference were checked. Body mass index (BMI) was estimated as weight in kilograms/height in meters (2). BMI was categorized using both WHO (15) and Asian (16) cutoff levels for comparison purposes. Blood pressure (BP) was measured by a standardized automated measurement system after the participant had been seated for 5 minutes. The mean of 2 readings was used for analysis.

After 12 hour of fasting, blood samples were taken in the morning. All blood samples were labeled and dispatched to the MRI laboratory and samples were centrifuged. Then serum samples were frozen without preservative at $\leq 20^{\circ} \mathrm{C}$ and sent to the laboratory of Mickagowa University, Japan for analysis in dry ice. Triglyceride (TG), fasting plasma sugar (FPG), high density lipoprotein (HDL), HbA1C and plasma insulin level were measured using an enzymatic colorimetric method with kits. New International Diabetes Federation definition was used to define MetS for this study (17). Metabolic syndrome was defined as presence of central obesity (defined as waist circumference $\geq 90 \mathrm{~cm}$ for male and $\geq 80 \mathrm{~cm}$ for female) plus any two of the following four factors. High triglycerides - TG $\geq 150 \mathrm{mg} / \mathrm{dl}$; low HDL cholesterol $-\mathrm{HDL}<40 \mathrm{mg} / \mathrm{dl}$ in males and $<50 \mathrm{mg} / \mathrm{dl}$ in females; high blood pressure - BP systolic $\geq 130 \mathrm{mmHg}$ or BP diastolic $\geq 85 \mathrm{mmHg}$; high fasting plasma glucose $\mathrm{FPG} \geq 100 \mathrm{mg} / \mathrm{dl}$.

Hyperinsulinaemia was defined as fasting plasma insulin level $\geq 10 \mu \mathrm{IU} / \mathrm{Ml}$ (18). Overweight, obesity, and high $\mathrm{HbA} 1 \mathrm{C}$ was defined as $\mathrm{BMI} \geq 25.0, \mathrm{BMI} \geq 30.0$ (15) and $\geq 6.5 \%$ respectively (19). Insulin resistance was assessed using the homeostasis model assessment (HOMA-IR) originally described by Mathew et al (20). HOMA-IR was calculated using the following formula: HOMA-IR $(\mathrm{mmol} / \mathrm{L} \times \geq \mu \mathrm{U} / \mathrm{ml})=$ fasting glucose $(\mathrm{mmol} / \mathrm{L}) \times$ fasting insulin $(\geq \mathrm{U} / \mathrm{ml}) / 22.5$. Insulin resistance was defined as HOMA-IR $>2.5$.

Study period was between February to June 2012. Ethical clearance was obtained from the ethical committee of Medical Research Institute. Differences were tested by using analysis of variance and chi square test; significance values were taken at 0.05 level. All data were analyzed by using SPSS for Windows, version 16.0 (SPSS Inc, Chicago).

\section{Results}

A total 345 study subjects were included in the study and response rate was $95.8 \%$. From total participants, 159 $(46.1 \%)$ were men and $186(53.9 \%)$ women. Table 1 shows 
that the prevalence of metabolic syndrome was $25.8 \%$ and there was no significant difference between men and women $(23.9 \%$ in men and $27.4 \%$ in women; $\mathrm{P}=0.27)$. It showed significantly increased prevalence of metabolic syndrome with increasing age in total sample, and women. The highest prevalence of metabolic syndrome was seen in age group 50-59 years in total sample, in men and women (35\%, 29\% and $41 \%$ respectively).

Prevalence of each component of MetS in studied population was: $62.9 \%$ for central obesity, $28.1 \%$ for high TG level, $35.9 \%$ for low HDL, $40 \%$ for high BP, $18.8 \%$ for high FPG, 3.9\% for high $\mathrm{HbA1C}, 26.9 \%$ for high insulin, $44.1 \%$ for overweight and $9.3 \%$ for obesity (Table 2 ). It showed increased prevalence of central obesity with increasing age. The prevalence of central obesity was significantly higher in women than in men $(77.4 \%$ vs $45.9 \%$; $\mathrm{P}=0.000$ ). Central obesity showed highest prevalence in age group 50-59 years in total sample, in men and women (68.3\%, 51.6\% and $85.2 \%$ respectively). High TG and high FPG was significantly more prevalent in men than women
(37.1\% vs 20.4\%; $\mathrm{P}=0.000$, and $27 \%$ vs $11.8 \% ; \mathrm{P}=0.000$ respectively). Conversely low HDL, overweight and obesity was significantly higher in women than men $(44.6 \%$ vs $25.8 \% ; \mathrm{P}=0.000,50.5 \%$ vs $36.5 \%: \mathrm{P}=0.006$ and $13.4 \%$ vs $4.4 \%: \mathrm{P}=0.003$ respectively). The highest prevalence of high BP, high FPG and high insulin was seen in age group of 50-59.

In total study subjects, $30.4 \%$ were normal (none of the MetS components were present), 30.1\% had one component, $18 \%$ two, $6.4 \%$ three, $2.9 \%$ four and $12.3 \%$ had five components of metabolic syndrome (Table 3 ).

Factors that can affect the prevalence of MetS are shown in Table 4. Among these factors, age, BMI, waist circumference, systolic BP, diastolic BP, FPG, HDL, TG, insulin, HbA1C and HOMA-IR were significantly higher in study subjects with MetS than without $(\mathrm{P}=0.00)$. Sex, area of residence and LDL level showed no significant differences between subjects with MetS and without MetS $(\mathrm{P}>0.05)$.

Table 1. Prevalence of metabolic syndrome according to sex and age group

\begin{tabular}{|c|c|c|c|}
\hline Age groups in years & $N$ & No. of MetS (\%) & Confidence interval (CI) \\
\hline $30-39$ & 16 & 15.1 & $11.3-18.9$ \\
\hline $40-49$ & 30 & 25.9 & $21.3-30.5$ \\
\hline $50-59$ & 43 & 35.0 & $29.9-40.0$ \\
\hline Total & 89 & 25.8 & 21.2-30.4 \\
\hline \multirow[t]{2}{*}{ Test Statistics } & & $\chi^{2}=10.5 ; \mathbf{P}=\mathbf{0 . 0 0 5}$ & \\
\hline & $\mathbf{M}$ & & \\
\hline $30-39$ & 8 & 16.0 & $10.3-21.7$ \\
\hline $40-49$ & 12 & 25.5 & $18.7-32.3$ \\
\hline $50-59$ & 18 & 29.0 & $21.9-36.1$ \\
\hline Total & 38 & $23.9^{1}$ & $17.3-30.5$ \\
\hline \multirow[t]{2}{*}{ Test Statistics } & & $\chi^{2}=2.9 ; \mathbf{P}=\mathbf{0 . 2 3}$ & \\
\hline & & & \\
\hline $30-39$ & 8 & 14.3 & $9.3-19.3$ \\
\hline $40-49$ & 18 & 26.1 & $19.8-32.4$ \\
\hline $50-59$ & 25 & 41.0 & $33.9-48.1$ \\
\hline Total & 51 & $27.4^{1}$ & 20.9-33.8 \\
\hline Test Statistics & & $\chi^{2}=9.2 ; \mathbf{P}=\mathbf{0 . 0 1}$ & \\
\hline
\end{tabular}

$\left(\chi^{2}=0.5 ; \mathbf{P}=\mathbf{0 . 2 7}\right)$ 
Table 2. Prevalence of each MetS component according to age and sex

\begin{tabular}{|c|c|c|c|c|c|c|c|c|c|}
\hline \multirow{2}{*}{$\begin{array}{l}\text { Age } \\
\text { In years }\end{array}$} & \multicolumn{8}{|c|}{ No. (\%) } & \multirow[b]{2}{*}{ Obese } \\
\hline & $\begin{array}{l}\text { Central } \\
\text { obesity }\end{array}$ & $\begin{array}{c}\text { High } \\
\text { TG }\end{array}$ & $\begin{array}{l}\text { Low } \\
\text { HDL }\end{array}$ & $\begin{array}{c}\text { High } \\
\text { BP }\end{array}$ & $\begin{array}{l}\text { High } \\
\text { FPG }\end{array}$ & $\begin{array}{c}\text { High } \\
\text { HbA1C }\end{array}$ & $\begin{array}{c}\text { High } \\
\text { insulin }\end{array}$ & $\begin{array}{l}\text { Over } \\
\text { weight }\end{array}$ & \\
\hline $30-39$ & $\begin{array}{r}57 \\
(53.8)\end{array}$ & $\begin{array}{r}28 \\
(26.4)\end{array}$ & $\begin{array}{r}37 \\
(34.9)\end{array}$ & $\begin{array}{r}20 \\
(18.9)\end{array}$ & $\begin{array}{r}9 \\
(8.5)\end{array}$ & $\begin{array}{r}3 \\
(3.6)\end{array}$ & $\begin{array}{r}27 \\
(25.7)\end{array}$ & $\begin{array}{r}42 \\
(39.6)\end{array}$ & $\begin{array}{r}9 \\
(8.5)\end{array}$ \\
\hline $40-49$ & $\begin{array}{r}76 \\
(65.5)\end{array}$ & $\begin{array}{r}33 \\
(28.4)\end{array}$ & $\begin{array}{r}46 \\
(39.7)\end{array}$ & $\begin{array}{r}48 \\
(41.4)\end{array}$ & $\begin{array}{r}24 \\
(20.7)\end{array}$ & $\begin{array}{r}4 \\
(4.2)\end{array}$ & $\begin{array}{r}27 \\
(23.7)\end{array}$ & $\begin{array}{r}52 \\
(44.8)\end{array}$ & $\begin{array}{r}12 \\
(10.3)\end{array}$ \\
\hline $50-59$ & $\begin{array}{r}84 \\
(68.3)\end{array}$ & $\begin{array}{r}36 \\
(29.3)\end{array}$ & $\begin{array}{r}41 \\
(33.3)\end{array}$ & $\begin{array}{r}70 \\
(56.9)\end{array}$ & $\begin{array}{r}32 \\
(26.0)\end{array}$ & $\begin{array}{r}4 \\
(4.0)\end{array}$ & $\begin{array}{r}38 \\
(30.9)\end{array}$ & $\begin{array}{r}58 \\
(47.2)\end{array}$ & $\begin{array}{r}11 \\
(8.9)\end{array}$ \\
\hline$P$ values & $\begin{array}{l}\chi^{2}=5.6 \\
P=0.06\end{array}$ & $\begin{aligned} \chi^{2} & =0.24 \\
\mathrm{P} & =0.89\end{aligned}$ & $\begin{array}{l}\chi^{2}=1.1 \\
P=0.56\end{array}$ & $\begin{array}{l}\chi^{2}=34.5 \\
\mathbf{P}=\mathbf{0 . 0 0 0}\end{array}$ & $\begin{array}{l}\chi^{2}=11.8 \\
\mathbf{P}=\mathbf{0 . 0 0 3}\end{array}$ & $\begin{array}{r}\chi^{2}=0.01 \\
P=0.6\end{array}$ & $\begin{array}{r}\chi^{2}=1.7 \\
\mathrm{P}=0.4\end{array}$ & $\begin{aligned} \chi^{2} & =1.4 \\
\mathrm{P} & =0.5\end{aligned}$ & $\begin{aligned} \chi^{2} & =0.3 \\
P & =0.9\end{aligned}$ \\
\hline \multicolumn{10}{|l|}{ Sex } \\
\hline Male & $\begin{array}{r}73 \\
(45.9)\end{array}$ & $\begin{array}{r}59 \\
(37.1)\end{array}$ & $\begin{array}{r}41 \\
(25.8)\end{array}$ & $\begin{array}{r}65 \\
(40.9)\end{array}$ & $\begin{array}{r}43 \\
(27.0)\end{array}$ & $\begin{array}{r}5 \\
(3.8)\end{array}$ & $\begin{array}{r}40 \\
(25.3)\end{array}$ & $\begin{array}{r}58 \\
(36.5)\end{array}$ & $\begin{array}{r}7 \\
(4.4)\end{array}$ \\
\hline Female & $\begin{array}{r}144 \\
(77.4)\end{array}$ & $\begin{array}{r}38 \\
(20.4)\end{array}$ & $\begin{array}{r}83 \\
(44.6)\end{array}$ & $\begin{array}{r}73 \\
(39.3)\end{array}$ & $\begin{array}{r}22 \\
(11.8)\end{array}$ & $\begin{array}{r}6 \\
(4.0)\end{array}$ & $\begin{array}{r}52 \\
(28.3)\end{array}$ & $\begin{array}{r}94 \\
(50.5)\end{array}$ & $\begin{array}{r}25 \\
(13.4)\end{array}$ \\
\hline$P$ values & $\begin{array}{l}\chi^{2}=36.5 \\
\mathbf{P}=\mathbf{0 . 0 0 0}\end{array}$ & $\begin{array}{l}\chi^{2}=11.7 \\
\mathbf{P}=\mathbf{0 . 0 0 0}\end{array}$ & $\begin{array}{l}\chi^{2}=13.2 \\
\mathbf{P}=\mathbf{0 . 0 0 0}\end{array}$ & $\begin{array}{r}\chi^{2}=0.09 \\
P=0.4\end{array}$ & $\begin{array}{l}\chi^{2}=12.9 \\
\mathbf{P}=\mathbf{0 . 0 0 0}\end{array}$ & $\begin{array}{r}\chi^{2}=0.00 \\
P=0.6\end{array}$ & $\begin{array}{r}\chi^{2}=0.4 \\
\mathrm{P}=0.3\end{array}$ & $\begin{array}{r}\chi^{2}=6.8 \\
\mathbf{P}=\mathbf{0 . 0 0 6}\end{array}$ & $\begin{array}{r}\chi^{2}=8.3 \\
\mathbf{P}=\mathbf{0 . 0 0 3}\end{array}$ \\
\hline Total & $\begin{array}{r}217 \\
(62.9)\end{array}$ & $\begin{array}{r}97 \\
(28.1)\end{array}$ & $\begin{array}{r}124 \\
(35.9)\end{array}$ & $\begin{array}{r}138 \\
(40.0)\end{array}$ & $\begin{array}{r}65 \\
(18.8)\end{array}$ & $\begin{array}{r}11 \\
(3.9)\end{array}$ & $\begin{array}{r}92 \\
(26.9)\end{array}$ & $\begin{array}{r}152 \\
(44.1)\end{array}$ & $\begin{array}{r}32 \\
(9.3)\end{array}$ \\
\hline \multicolumn{10}{|c|}{ Male } \\
\hline $30-39$ & $\begin{array}{r}19 \\
(38.0)\end{array}$ & $\begin{array}{r}20 \\
(40.0)\end{array}$ & $\begin{array}{r}11 \\
(22.0)\end{array}$ & $\begin{array}{r}13 \\
(26.0)\end{array}$ & $\begin{array}{r}6 \\
(12.0)\end{array}$ & $\begin{array}{r}5 \\
(3.2)\end{array}$ & $\begin{array}{r}9 \\
(18.0)\end{array}$ & $\begin{array}{r}16 \\
(32.0)\end{array}$ & $\begin{array}{r}2 \\
(4.0)\end{array}$ \\
\hline $40-49$ & $\begin{array}{r}22 \\
(46.8)\end{array}$ & $\begin{array}{r}19 \\
(40.4)\end{array}$ & $\begin{array}{r}15 \\
(31.9)\end{array}$ & $\begin{array}{r}21 \\
(44.7)\end{array}$ & $\begin{array}{r}15 \\
(31.9)\end{array}$ & $\begin{array}{r}5 \\
(3.2)\end{array}$ & $\begin{array}{r}13 \\
(28.3)\end{array}$ & $\begin{array}{r}18 \\
(38.3)\end{array}$ & $\begin{array}{r}1 \\
(2.1)\end{array}$ \\
\hline $50-59$ & $\begin{array}{r}32 \\
(51.6)\end{array}$ & $\begin{array}{r}20 \\
(32.3)\end{array}$ & $\begin{array}{r}1 \\
(24.2)\end{array}$ & $\begin{array}{r}31 \\
(50.0)\end{array}$ & $\begin{array}{r}22 \\
(35.5)\end{array}$ & $\begin{array}{r}1 \\
(1.9)\end{array}$ & $\begin{array}{r}18 \\
(29.0)\end{array}$ & $\begin{array}{r}24 \\
(38.7)\end{array}$ & $\begin{array}{r}4 \\
(6.5)\end{array}$ \\
\hline $\begin{array}{l}\text { Test } \\
\text { statistics }\end{array}$ & $\begin{array}{l}\chi^{2}=2.1 \\
\mathrm{P}=0.35\end{array}$ & $\begin{array}{l}\chi^{2}=1.0 \\
\mathrm{P}=0.59\end{array}$ & $\begin{aligned} \chi^{2} & =1.4 \\
\mathrm{P} & =0.5\end{aligned}$ & $\begin{array}{l}\chi^{2}=6.9 \\
\mathbf{P}=\mathbf{0} .03\end{array}$ & $\begin{array}{l}\chi^{2}=8.5 \\
\mathbf{P}=\mathbf{0 . 0 1}\end{array}$ & $\begin{array}{r}\chi^{2}=0.04 \\
P=0.9\end{array}$ & $\begin{array}{r}\chi^{2}=2.1 \\
\mathrm{P}=0.4\end{array}$ & $\begin{aligned} \chi^{2} & =0.6 \\
P & =0.7\end{aligned}$ & $\begin{array}{r}\chi 2=1.2 \\
\mathrm{P}=0.5\end{array}$ \\
\hline \multicolumn{10}{|c|}{ Female } \\
\hline $30-39$ & $\begin{array}{r}38 \\
(67.9)\end{array}$ & $\begin{array}{r}8 \\
(14.3)\end{array}$ & $\begin{array}{r}26 \\
(46.4)\end{array}$ & $\begin{array}{r}7 \\
(12.5)\end{array}$ & $\begin{array}{r}3 \\
(5.4)\end{array}$ & $\begin{array}{r}1 \\
(2.2)\end{array}$ & $\begin{array}{r}18 \\
(32.7)\end{array}$ & $\begin{array}{r}26 \\
(46.4)\end{array}$ & $\begin{array}{r}7 \\
(12.5)\end{array}$ \\
\hline $40-49$ & $\begin{array}{r}54 \\
(78.3)\end{array}$ & $\begin{array}{r}14 \\
(20.3)\end{array}$ & $\begin{array}{r}31 \\
(44.9)\end{array}$ & $\begin{array}{r}27 \\
(39.1)\end{array}$ & $\begin{array}{r}9 \\
(13.0)\end{array}$ & $\begin{array}{r}2 \\
(3.5)\end{array}$ & $\begin{array}{r}14 \\
(20.6)\end{array}$ & $\begin{array}{r}34 \\
(49.3)\end{array}$ & $\begin{array}{r}11 \\
(15.9)\end{array}$ \\
\hline $50-59$ & $\begin{array}{r}52 \\
(85.2)\end{array}$ & $\begin{array}{r}15 \\
(26.2)\end{array}$ & $\begin{array}{r}26 \\
(42.6)\end{array}$ & $\begin{array}{r}39 \\
(63.9)\end{array}$ & $\begin{array}{r}10 \\
(16.4)\end{array}$ & $\begin{array}{r}3 \\
(6.4)\end{array}$ & $\begin{array}{r}20 \\
(32.8)\end{array}$ & $\begin{array}{r}34 \\
(55.7)\end{array}$ & $\begin{array}{r}7 \\
(11.5)\end{array}$ \\
\hline $\begin{array}{l}\text { Test } \\
\text { statistics }\end{array}$ & $\begin{array}{l}\chi^{2}=5.1 \\
P=0.08\end{array}$ & $\begin{array}{l}\chi^{2}=2.6 \\
P=0.28\end{array}$ & $\begin{aligned} \chi^{2} & =1.8 ; \\
P & =0.9\end{aligned}$ & $\begin{array}{l}\chi^{2}=32.4 \\
\mathbf{P}=\mathbf{0 . 0 0 0}\end{array}$ & $\begin{array}{r}\chi^{2}=3.6 \\
P=0.2\end{array}$ & $\begin{array}{l}\chi^{2}=1.1 \\
P=0.58\end{array}$ & $\begin{array}{r}\chi^{2}=3.1 \\
\mathrm{P}=0.2\end{array}$ & $\begin{aligned} \chi^{2} & =1.2 \\
\mathrm{P} & =0.5\end{aligned}$ & $\begin{aligned} \chi^{2} & =0.6 \\
P & =0.7\end{aligned}$ \\
\hline
\end{tabular}

$($ Overweight $=\mathrm{BMI} \geq 25.0$ and Obesity $=\mathrm{BMI} \geq 30.0)$ 
Table 3. Prevalence of metabolic syndrome components and insulin resistance in studied population

\begin{tabular}{lrcc}
\hline No. of components & No. & $\%$ & Confidence interval (CI) \\
\hline No any component & 105 & 30.4 & $25.6-35.3$ \\
1 component & 104 & 30.1 & $25.3-34.9$ \\
2 components & 62 & 18.0 & $14.0-22.1$ \\
3 components & 22 & 6.4 & $3.8-8.9$ \\
4 components & 10 & 2.9 & $1.1-4.7$ \\
5 components & 42 & 12.3 & $8.8-15.8$ \\
Insulin resistance (HOMA-IR) & 342 & 22.3 & $19.8-28.9$ \\
Total & $\mathbf{3 4 5}$ & $\mathbf{1 0 0 . 0}$ & \\
\hline
\end{tabular}

Table 4. Relationship of MetS with associated factors

\begin{tabular}{cccc}
\hline Associated factors & No. (\%) with MetS & Total & Pvalue
\end{tabular}

Age groups in years

$\begin{array}{rrrrr}30-39 & 16(18.0) & 90(35.2) & 106 & \chi^{2}=11.7 \\ 40-49 & 30(33.7) & 86(33.6) & 116 & \mathbf{P}=\mathbf{0 . 0 0 3} \\ 50-59 & 43(48.3) & 80(31.3) & 123\end{array}$

Sex

\begin{tabular}{|c|c|c|c|c|}
\hline Male & $38(42.7)$ & $121(47.3)$ & 159 & $\chi^{2}=0.5$ \\
\hline Female & $51(57.3)$ & $135(52.7)$ & 186 & $\mathrm{P}=0.27$ \\
\hline
\end{tabular}

Area of residence

\begin{tabular}{|c|c|c|c|c|}
\hline Urban & $41(46.1)$ & $135(52.7)$ & 176 & $\chi^{2}=1.8$ \\
\hline Rural & $48(53.9)$ & $121(47.3)$ & 169 & $\mathrm{P}=0.17$ \\
\hline \multicolumn{5}{|l|}{ BMI $\left(\mathrm{kg} / \mathrm{m}^{2}\right)$} \\
\hline$<18.5$ & $1(1.1)$ & $21(8.2)$ & 22 & \multirow{4}{*}{$\begin{array}{c}\chi^{2}=23.1 \\
\mathbf{P}=\mathbf{0 . 0 0 0}\end{array}$} \\
\hline $18.5-24.9$ & $30(33.7)$ & $140(54.7)$ & 170 & \\
\hline 25.0-29.9 & $44(49.4)$ & $76(29.7)$ & 120 & \\
\hline$\geq 30.0$ & $14(15.7)$ & $19(7.4)$ & 33 & \\
\hline \multicolumn{5}{|l|}{ BMI Asian cut-off values $\left(\mathrm{kg} / \mathrm{m}^{2}\right)$} \\
\hline$<18.5$ & $1(1.1)$ & $21(8.2)$ & 22 & \multirow{5}{*}{$\begin{array}{l}\chi^{2}=23.2 \\
\mathbf{P}=\mathbf{0 . 0 0 0}\end{array}$} \\
\hline $18.5-22.9$ & $16(18.0)$ & $84(33.8)$ & 100 & \\
\hline $23.0-24.9$ & $14(15.7)$ & $56(21.9)$ & 70 & \\
\hline$\geq 25.0$ & $58(65.2)$ & $95(37.1)$ & 153 & \\
\hline \multirow[t]{2}{*}{ Total } & $89(25.8)$ & $256(74.2)$ & 345 & \\
\hline & \multicolumn{3}{|c|}{ Mean (SD) } & \\
\hline $\mathrm{BMI}\left(\mathrm{kg} / \mathrm{m}^{2}\right)$ & $26.4(3.9)$ & $23.9(4.0)$ & $24.6(4.1)$ & $F=; P=0.000$ \\
\hline Waist circumference $(\mathrm{cm})$ & $94.2(7.9)$ & $85.3(10.2)$ & $87.6(10.4)$ & $F=; P=0.000$ \\
\hline FPG (mg/l) & $102.6(28.7)$ & $91.7(22.9)$ & $94.5(24.9)$ & $F=; P=0.000$ \\
\hline $\mathrm{TG}(\mathrm{mg} / \mathrm{l}) 170.4(73.6)$ & $110.1(61.5)$ & $125.6(69.9)$ & $F=; P=0.000$ & \\
\hline $\mathrm{HDL}(\mathrm{mg} / \mathrm{l})$ & $44.5(10.0)$ & $50.3(10.1)$ & $48.8(10.3)$ & $F=; P=0.000$ \\
\hline $\mathrm{LDL}(\mathrm{mg} / \mathrm{l})$ & $153.5(37.9)$ & $144.6(35.9)$ & $146.9(36.5)$ & $F=; P=0.05$ \\
\hline Systolic BP (mmHg) & $136.8(14.6)$ & $121.8(15.9)$ & $125.7(16.9)$ & $F=; P=0.000$ \\
\hline Diastolic BP (mmHg) & $81.1(11.0)$ & $72.1(11.0)$ & $74.4(11.7)$ & $F=; P=0.000$ \\
\hline HbA1C level $(\%)(n=279)$ & $4.3(1.4)$ & $3.9(1.2)$ & $4.0(1.2)$ & $F=; P=0.01$ \\
\hline Plasma insulin level $(\mu \mathrm{IU} / \mathrm{mL})$ & $10.7(10.5)$ & $6.9(4.4)$ & $7.9(6.7)$ & $F=; P=0.000$ \\
\hline HOMA-IR (n=342) & $2.72(2.8)$ & $1.65(1.9)$ & $1.93(2.2)$ & $F=; P=0.000$ \\
\hline
\end{tabular}


Table 5 shows that prevalence of hyperinsulinaemia was $26.9 \%$ and no significant difference between men and women (25.3\% in men and $28.3 \%$ in women). The highest prevalence of hyperinsulinaemia was seen in age group 50-59 years (30.9\%). The prevalence of hyperinsulinaemia was significantly higher in study subjects living in rural sector $(33.1 \%$ vs $21 \% ; \mathrm{P}=0.01)$, having metabolic syndrome ( $46.6 \%$ vs $20.1 \% ; \mathrm{P}=0.000)$, central obesity ( $33 \%$ vs $16.5 \%$; $=0.001)$, high $\mathrm{BP}(26.2 \%$ vs $20.6 \% ; \mathrm{P}=0.001)$, low HDL ( $34.7 \%$ vs $22.6 \% ; \mathrm{P}=0.01$ ) and high TG $(34.4 \%$ vs $24 \%$; $=0.04)$. The highest prevalence of hyperinsulinaemia was seen in obese subjects according to both WHO and Asian classification (42.4\% and $36.8 \%$ respectively).

Prevalence of insulin resistance was $22.3 \%$ and significantly higher in study subjects with metabolic syndrome (44.3\% vs $14.6 \% ; \mathrm{P}=0.000)$, obesity $(33.3 \%$ vs $31.1 \%$ and $16.1 \% ; \mathrm{P}=0.002)$, central obesity $(26.5 \% \mathrm{vs}$ $15.1 \% ; \mathrm{P}=0.009)$, high $\mathrm{BP}(30.7 \%$ vs $16.7 \% ; \mathrm{P}=0.002)$ and high TG $(33.3 \%$ vs $18 \% ; \mathrm{P}=0.002)$ as shown in Table 5.

Table 5. Prevalence of hyperinsulinaemia and insulin resistance in MetS associated factors

\begin{tabular}{|c|c|c|c|c|c|c|}
\hline \multirow[t]{2}{*}{ Associated factors } & \multirow[t]{2}{*}{ Total } & \multicolumn{2}{|c|}{ Hyperinsulinaemia } & \multirow[b]{2}{*}{$P$ value } & \multicolumn{2}{|c|}{ HOMA-LR } \\
\hline & & No. (\%) & $95 \% C I$ & & No. (\%) & P value \\
\hline \multicolumn{7}{|l|}{ Age groups in years } \\
\hline $30-39$ & 105 & $27(25.7)$ & $21.7-30.3$ & $\chi^{2}=1.7$ & $19(18.1)$ & $\chi^{2}=2.1$ \\
\hline $40-49$ & 114 & $27(23.7)$ & $19.2-28.2$ & $\mathrm{P}=0.43$ & $25(21.9)$ & $\mathrm{P}=0.3$ \\
\hline $50-59$ & 123 & $38(30.9)$ & $26.0-35.8$ & & $32(26.2)$ & \\
\hline \multicolumn{7}{|l|}{ Sex } \\
\hline Male & 158 & $40(25.3)$ & $20.7-29.9$ & $\chi^{2}=0.38$ & $35(22.3)$ & $\chi^{2}=0.0$ \\
\hline Female & 184 & $52(28.3)$ & $23.5-33.1$ & $\mathrm{P}=0.3$ & $41(22.3)$ & $\mathrm{P}=0.5$ \\
\hline \multicolumn{7}{|l|}{ Area of residence } \\
\hline Urban & 176 & $37(21.0)$ & $16.7-25.3$ & $\chi^{2}=6.4$ & $31(17.7)$ & $\chi^{2}=4.3$ \\
\hline Rural & 166 & $55(33.1)$ & 28.1-38.1 & $\mathrm{P}=\mathbf{0 . 0 0 8}$ & $45(27.1)$ & $P=0.03$ \\
\hline \multicolumn{7}{|l|}{ Metabolic syndrome } \\
\hline Yes & 88 & $41(46.6)$ & $41.3-51.9$ & $\chi^{2}=23.4$ & $39(44.3)$ & $\chi^{2}=33.2$ \\
\hline No & 254 & $51(20.1)$ & $15.9-24.4$ & $P=0.000$ & $37(14.6)$ & $\mathbf{P}=\mathbf{0 . 0 0 0}$ \\
\hline \multicolumn{7}{|l|}{ BMI (kg/m²) } \\
\hline$<18.5$ & 21 & $1(4.8)$ & $2.5-7.1$ & $\chi^{2}=17.7$ & $1(4.8)$ & $\chi^{2}=15.1$ \\
\hline $18.5-24.9$ & 169 & $35(20.7)$ & $16.4-24.9$ & $P=0.001$ & $27(16.1)$ & $\mathrm{P}=0.002$ \\
\hline $25.0-29.9$ & 119 & $42(35.3)$ & $30.2-40.4$ & & $37(31.1)$ & \\
\hline$\geq 30.0$ & 33 & $14(42.4)$ & $37.2-47.6$ & & $11(33.3)$ & \\
\hline \multicolumn{7}{|l|}{ BMI Asian cut-off } \\
\hline$<18.5$ & 21 & $1(4.8)$ & $2.5-7.1$ & $\chi^{2}=17.7$ & $1(4.8)$ & $\chi^{2}=16.1$ \\
\hline $18.5-22.9$ & 99 & $17(17.2)$ & $13.2-21.2$ & $\mathrm{P}=0.001$ & $13(13.3)$ & $P=0.001$ \\
\hline $23.0-24.9$ & 70 & $18(25.7)$ & $21.1-30.3$ & & $14(20.0)$ & \\
\hline$\geq 25.0$ & 152 & $56(36.8)$ & $31.7-41.9$ & & $48(31.6)$ & \\
\hline \multicolumn{7}{|l|}{ Central obesity } \\
\hline Yes & 215 & $71(33.0)$ & $28.0-37.9$ & $\chi^{2}=11.0$ & $57(26.5)$ & $\chi^{2}=5.9$ \\
\hline No & 127 & $21(16.5)$ & $12.6-20.4$ & $P=0.001$ & $19(15.1)$ & $P=0.009$ \\
\hline \multicolumn{7}{|l|}{ High BP } \\
\hline Yes & 138 & $50(26.2)$ & $31.1-41.3$ & $\chi^{2}=17.7$ & $42(30.7)$ & $\chi^{2}=9.2$ \\
\hline No & 204 & $42(20.6)$ & $16.3-24.9$ & $\mathrm{P}=0.001$ & $34(16.7)$ & $P=0.002$ \\
\hline \multicolumn{7}{|l|}{ LowHDL } \\
\hline Yes & 121 & $42(34.7)$ & $29.7-39.7$ & $\chi^{2}=17.7$ & $32(26.7)$ & $\chi^{2}=2.1$ \\
\hline No & 221 & $50(22.6)$ & $18.2-27.0$ & $\mathbf{P}=\mathbf{0 . 0 1}$ & $44(19.9)$ & $P=0.09$ \\
\hline \multicolumn{7}{|l|}{ High TG } \\
\hline Yes & 96 & $33(34.4)$ & 29.4-39.4 & $\chi^{2}=3.8$ & $32(33.3)$ & $\chi^{2}=9.4$ \\
\hline No & 246 & $59(24.0)$ & $19.5-28.5$ & $P=0.04$ & $44(18.0)$ & $P=0.002$ \\
\hline Total & 342 & $92(26.9)$ & $22.2-31.6$ & & $76(22.3)$ & \\
\hline
\end{tabular}




\section{Discussion}

With the metabolic syndrome driving the twin global epidemics of type 2 diabetes and CVD there is an overwhelming moral, medical and economic imperative to identify those individuals with metabolic syndrome early, so that lifestyle interventions and treatment may prevent the development of diabetes and or CVDs (5). The results of this study showed that the prevalence of metabolic syndrome is high in studied population and it increases with increment of age and BMI significantly. The prevalence of MetS was $25.8 \%$ in this study which is lower than previous studies in Sri Lanka (27.1\%) (12). This may be due to methodological and sampling differences between these studies. In DECODE study that was conducted in 9 European countries, MetS was detected in $32 \%$ of men and $28.5 \%$ of women (21). The prevalence of MetS has been reported in other parts of the world with different diagnostic criteria made the comparison difficult.

Prevalence of high BP (40\%), high FPG (18.8\%), overweight (44.1\%) and obesity (9.3\%) was higher in our sample than the prevalence estimated by the WHO in 2008 (39.2\%, 8.8\%, 21.9\% and 5.1\%) (14). However, high BP was lower $(40 \%)$ in this study than the previous national study in $2006(81.1 \%)$ (12). Prevalence of high TG in our study was $28.1 \% ; 37.1 \%$ in men and $20.4 \%$ in women but in previous study it was $55.1 \%$ (12), in Sri Lanka. It may be due to different criteria used to define the components of MetS in these studies.

Relationship between associated factors and MetS showed very useful information for future actions. Our study identified that age, BMI, central obesity, FPG, TG, HDL, LDL, systolic BP, diastolic BP, HbA1C, plasma insulin, HOMA-I had significant relationship with MetS, but not with the area of residence. It was identified in some studies that many factors including: age, ethnicity, weight, menopause in women, smoking, low income economies, high carbohydrate intake, and low physical activity play a role in metabolic syndrome (22).

In most studies $(23,24)$ increasing age was the key factor affecting the prevalence of metabolic syndrome and it was also shown in our study. Increasing in BMI was correlated with increasing prevalence of MetS, hyperinsulinaemia and insulin resistance in this study. This is in agreement with other studies (9). In this study, prevalence of metabolic syndrome was significantly higher in women than men as shown in other studies $(12,23)$. This may be due to reduction of physical activity in Sri Lankan women which may cause higher rate of central obesity and low HDL. Strengths of our study was that it is a community based study including both urban and rural population of Sri Lanka. However, there was no difference of prevalence of MetS between urban and rural populations but rural subjects had significantly high level of insulin resistance. One limitation was that, this study was cross sectional that does not allow us to draw any causal interference which indicates the need of a longitudinal study.

Nearly one third of our sample did not have any component of MetS which is lower than the previously reported figure (51\%) (13). Presence of two or more components were noted in $27.3 \%$ of our sample compared to the previous study (25\% between $20-40$ years) (13). This information is very useful to make decision on appropriate population screening to prevent MetS in the future.

Prevalence of hyperinsulinaemia in our study was $26.9 \%, 25.3 \%$ in men and $28.3 \%$ in women and insulin resistance was $22.3 \%$. Living in rural area, presence of metabolic syndrome, obesity, central obesity, high BP and high TG were strongly associated with both hyperinsulinaemia and insulin resistance.

Studies in the general population revealed that hyperinsulinemiais an early feature, of the cardiovascular clusters of MetS. It a surrogate marker to predict future development of MetS, but also the increased risk for future CVD as well (25). Therefore the results of our study strongly emphasise the urgent need of implementing lifestyle modifications for this population which help in reversing hyperinsulinaemia and insulin resistance to prevent MetS and CVD in the future.

\section{Conclusions}

Metabolic syndrome has high prevalence in our population and its prevalence increases with increasing age and BMI. Women are at higher risk for MetS than men. Metabolic syndrome was strongly associated with hyperinsulinaemia and insulin resistance. Strategies to reverse hyperinsulinaemia to reduce the risk in developing type-2 diabetes and cardiovascular diseases need to be identified.

\section{Acknowledgement}

We would like to thank medical officers of health and staff of Kotte, Kollonnawa and Aththanagalla for assisting us in collecting data and samples, staff of the Department of Nutrition, Medical Research Institute in collecting data and samples.

\section{References}

1. Kassi E, Pervanidou P, Kaltsas G, Chrousos G. Metabolic syndrome: definitions and controversies. BMC Medicine 2011; 9: 48.

2. Alberti KG, Zimmet P, Shaw J. IDF Epidemiology Task Force Consensus Group. The metabolic syndrome new worldwide definition. Lancet 2005; 366: 1059-62. 
3. Alberti KG, Zimmet P, Shaw J. Metabolic syndrome - a new world-wide definition. A Consensus Statement from the International Diabetes Federation. Diabet Med 2006; 23(5): 469-80.

4. Stern M, Williams K, Gonzalez-Villalpando C, et al. Does the metabolic syndrome improve identification of individuals at risk of type 2 diabetes and/or cardiovascular disease? Diabetes Care 2004; 27(11): 2676-81.

5. Reaven GM. Banting lecture 1988. Role of insulin resistance in human disease. Diabetes 1988; 37: 1595-1607.

6. Kaplan NM. The deadly quartet. Upper-body obesity, glucose intolerance, hypertriglyceridemia, and hypertension. Arch Intern Med 1989; 49: 1514-20.

7. DeFronzo RA, Ferrannini E. Insulin resistance. A multifaceted syndrome responsible for NIDDM, obesity, hypertension, dyslipidemia, and atherosclerotic cardiovascular disease. Diabetes Care 1991; 14: 173-94.

8. Fujioka S, Matsuzawa Y, Tokunaga K, Tarui S. Contribution of intra-abdominal fat accumulation to the impairment of glucose and lipid metabolism in human obesity. Metabolism 1987; 36: 54-9.

9. Onat A, Ugur M, Can G, Yuksel H, Hergenc G. Visceral adipose tissue and body fat mass: predictive values for and role of gender in cardiometabolic risk among Turks. Nutrition 2010; 26: 382-89.

10. Ramachandran A, Snehalatha C, Satyavani K, Sivasankari $\mathrm{S}$, Vijay V. Metabolic syndrome in urban Asian Indian adults - a population study using modified ATP III criteria. Diabetes Res Clin Pract 2003; 60(3): 199-204.

11. Shahbazian et al. Metabolic syndrome and its correlated factors in an urban population in South West of Iran. Journal of Diabetes and Metabolic Disorders 2013; 12: 11.

12. Katulanda et al. Metabolic syndrome among Sri Lankan adults: prevalence, patterns and correlates. Diabetology and Metabolic Syndrome 2012; 4: 24.

13. Wijesuriya M, Gulliford M, Charlton J, Vasantharaja L, Viberti G, et al. High Prevalence of Cardio-Metabolic Risk Factors in a Young Urban Sri-Lankan Population. PLOS ONE 2012; 7(2): e31309.

14. World Health Organisation: NCD country profiles. World Health Organisation, Geneva; 2011.
15. World Health Organisation: Physical status: the use and interpretation of anthropometry. World Health Organisation Geneva; 1995.

16. WHO expert consultation. Appropriate body mass index for Asian population and its implication for policy and intervention strategies. The Lancet 2004; 157-63.

17. International Diabetes Federation. The IDF consensus worldwide definition of the metabolic syndrome. International Diabetes Federation, Brussels; 2006.

18. British Medical Journal. Best practices. 2013. http:// bestpractice.bmj.com/best-practice/monograph/1137.html.

19. World Health Organisation: Use of Glycated Haemoglobin (HbA1C) in the Diagnosis of Diabetes Mellitus. Abbreviated report of WHO consultation. Geneva. 2011.

20. Matthews DR, Hosker JP, Rudenski AS, Naylor BA, Treacher DF, Turner RC. Homeostasis model assessment: insulin resistance and $\beta$-cell function from fasting plasma glucose and insulin concentrations in man. Diabetologia 1985; 28: 412-19.

21. Qiao Q, Pitkaniemi J, Tuomilehto J, et al. Comparison of different definition of the metabolic syndrome in relation to cardiovascular mortality in European men and women. Diabetology 2006; 49(12): 2837-46.

22. Park YW, Zhu S, Palaniappan L, et al: The metabolic syndrome: prevalence and associated risk factors finding in the US population from the Third National Health and Nutrition Examination Survey, 1998-1994. Arch Intern Med. 2003; 163(4): 427-36.

23. Sadrbafoghi SM, Salar M, Rafiee M, et al: Prevalence and criteria of metabolic syndrome in an urban population: Yazd healthy heart project. Tehran Univ. Med. J 2007; 64(10): 90-6.

24. Zaliunas R, Slapikas R, Luksiene D, et al. Prevalence of metabolic syndrome components in patients with acute coronary syndromes. Medicina (Kaunas) 2008; 44(3): $182-8$.

25. Sung KC, Seo MH, Rhee EJ, Wilson AM. Elevated fasting insulin predicts the future incidence of metabolic syndrome: a 5 year follow-up study. Cardiovascular Diabetology 2011; 10: 108. 